\title{
Developments in Multidimensional Item Response Theory
}

\author{
Terry Ackerman, Guest Editor \\ University of Illinois, Champaign-Urbana
}

This Special Issue of APM is devoted to current developments in multidimensional item response theory (MIRT). It consists of eight papers that present applications of MIRT models to practical testing problems. Six are in this issue and two will be published in the March 1997 issue. The goal of this Special Issue is to provide the testing community with innovative MIRT techniques that can be used to better comprehend and explain the assessment process. It is hoped that measurement specialists will use these psychometric tools to gain greater insight into what their tests are measuring and subsequently create more valid and reliable instruments.

The first paper by Ackerman introduces different ways to examine MIRT analyses graphically. Focusing primarily on the two-dimensional compensatory MIRT model, graphical examples include item response surfaces, information vectors, centroid plots of conditional latent trait distributions, and pictorial representation of maximum likelihood estimation of trait level in a two-dimensional latent trait space.

Stout, Roussos, and Nandakumar guide the testing practitioner through analgorithmic approach to assessing the dimensionality of-item response data. Several different analytic procedures including DIMTEST, HCA, PROX, and DETECT developed by Stout and his students are discussed. Examples of these procedure on real data are provided.

Intimately tied to the issue of dimensionality is the issue of differential item functioning (DIF). The third paper by Roussos and Stout identifies a multidimensional-based guide to detect DIF. This new paradigm unifies the substantive and statistical DIF approaches using a mathematically rigorous conceptualization of DIF. The authors encourage greater integration of DIF analyses with the overall test development process.

The next paper by van der Linden further examines test construction methodology from a multidimensional perspective. Several cases of multidimensional test assembly that utilize a linear programming model are reviewed.

One of the applications of item response theory (IRT) to practical testing problems is computer adaptive testing (CAT). Luecht describes how CAT can be extended to the multidimensional case. A heuristic optimization framework is outlined and extensions to both univariate and multivariate statistical objective functions with such constraints as content balancing are discussed. Implications for certification and licensure exams are addressed.

An important problem that practitioners using multidimensional item parameters must resolve is how to link estimates from different MIRT calibration runs. Davey, Oshima, and Lee summarize the issues surrounding proper transformations that map separate calibrations to a common metric. Extensions of unidimensional linking procedures to multidimensional latent trait models are explored.

Adams, Wilson, and Wang discuss the multidimensional random coefficients multinomial logit model. This model generalizes a wide range of Rasch models to the multidimensional case, including the simple 
logistic model, Master's partial credit model, Wilson's ordered partition model, and Fisher's linear logistic model. Other models discussed are Anderson's multidimensional Rasch model for repeated testing and Embretson's multidimensional modeling for learning and change. The final paper, by Reckase, traces the history of multidimensional IRT modeling and provides a preview to future directions and applications of MIRT models.

Testing practitioners are encouraged to use these seminal articles as stepping stones to advance multidimensional modeling and thereby develop a better understanding of the interaction between test items and examinee traits. 